\title{
Facial Affect Detection using Transfer Learning: A Comparative Study
}

\author{
Hardika Patel a, Harshil Pandya a, Dhananjay Theckedath ${ }^{\text {, }}$, Rithesh Kini b \\ a. Research Assistant, Biomedical Dept, Thadomal Shahani College of Engineering \\ b. Assistant Professor, Biomedical Dept, Thadomal Shahani College of Engineering
}

\begin{abstract}
Facial affect analysis is perceived as one of the most complex and challenging areas for humanisation of robots. Several Facial Expression Recognition (FER) systems apply generic machine learning algorithms to extract facial features. This results in an erroneous classification of previously unseen data. This paper improvises on previous research done on emotion detection and implements techniques to leverage the potential of Convolutional Neural Networks (CNNs) effectively to classify a set of grayscale images of human faces into seven distinct emotion categories. We experiment with some popular transfer learning models to achieve a maximum accuracy of $98 \%$ for the seven-class classification task. To eke out further precision and reduce the value loss, we incorporate the Squeeze and Excitation Network to the ResNet-50 model, which resulted in a validation accuracy of $99.36 \%$.
\end{abstract}

Index Terms - Expression recognition, Emotion Classification, SENet, Deep Neural Networks

\section{INTRODUCTION}

$\mathrm{E}$ MOTIONS share an inextricable link with human cognition and perception, thereby playing a significant role in decision making, communicating and maintaining social relationships. Hence, a foundational strategy to decrease the communicative differences between a human and a computer would be to incorporate in it, the ability to accurately categorise and respond to the affective states of the human operator.

In spite of being one of the least emphasised topic in the domain of artificial intelligence, facial affect detection demonstrates essential ramifications in the implementation of human-computer interface projects. It is widely believed that any system that is conditioned to perceive and respond to the operator's emotion during an interaction can improve the quality of interaction. This makes the computer interface more user-friendly, interactive and viable. Moreover, it might enhance the ability of a computer to make decisions and choices for a given task.

In this project, we have trained a convolutional neural network (CNN) to recognise and classify seven facial expressions: anger, contempt, disgust, fear, happy, surprise and sad. Face detection is performed using the Viola-Jones Algorithm in MATLAB while the CNN performs feature extraction and classification. The algorithm was trained on images from the Cohn-Kanade AU-Coded Expression Database Ver- sion $2(\mathrm{CK}+)$, which includes labelled images of 593 sequences from 123 posers, aged 18-50 years, and was recorded in settings controlled for illumination and angle. Three CNNs were trained using various transfer learning models for improvement in training time and accuracy (namely VGG16, ResNet-50, SE-ResNet 50).

In the following sections of this paper, we will elaborate on our problem statement, present an overview of transfer learning and the models used, and a report of our work.

\section{Problem Statement}

The objective of this project is to anticipate the emotion of a facial expression from a given grayscale picture of a person's face. Our evaluation metric will be the accuracy for each emotion (fraction of correctly classified images), supplemented by a confusion matrix that highlights which emotions are better recognized than others. In short,

- Input: $256 \times 256$ grayscale image of a face

- Output: Emotion conveyed by facial expression

\section{SCOPE OF THE PROJECT}

The practical goal of developing an affect detection algorithm is to augment the quality of human-computer interaction by endowing computers with the potential to recognise and respond suitably to human emotions. Its application ranges from its vitality in clinical psychology to pain assessment.

\section{Classification OF EMOTIONS}

Categorising the emotions can be a challenging issue as there is a lack of consensus, among various researchers, about the existence of accurate or universal standardised emotions.

Most present-day endeavours to recognise facial expression are formulated using the Facial Action Coding System (FACS) of psychologist Paul Ekman [1], which parameterised the facial behaviour based on the action of a muscle. This set of parameters are then decoded to generate various facial expressions.

For simplicity, we concentrate on the categorisation of facial affect into seven elementary emotions (anger, contempt, disgust, fear, happiness, sadness and surprise) as elucidated in the research done by Ekman and Friesen. [2][11] 
V. DATASeT

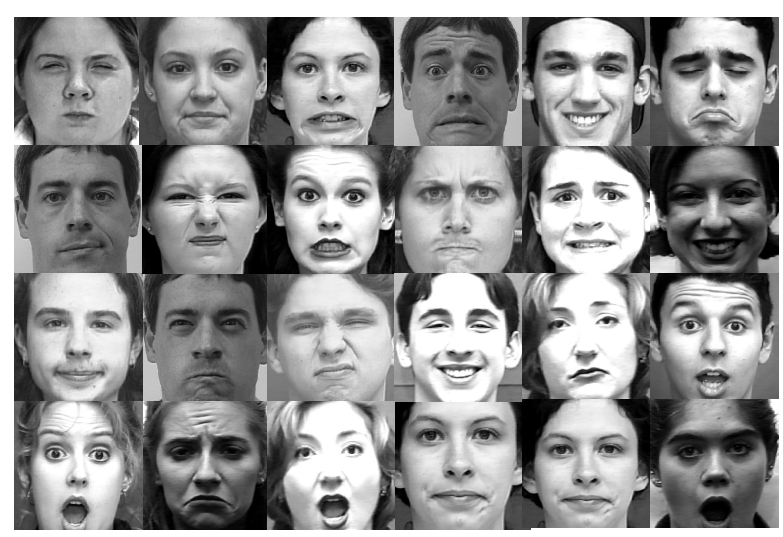

Fig. 1. Sample images from the final modified Cohn Kanade Database.

\section{A. Cohn-Kanade Database}

The Cohn-Kanade AU-Coded Expression Database Version $2(\mathrm{CK}+)[3]$ is an outstanding dataset of 593 recordings (10,708 frames) comprising of posed and non-posed sequences, which decode for their respective action units and their corresponding emotions. The database maintains a diversity among the subjects in terms of age, gender and race. While aged between 18 to 50 years, the dataset contains $31 \%$ male, $81 \%$, Euro-American, $13 \%$ Afro-American, and $6 \%$ other groups. A standardised lab setting with controlled light conditions and head motion is prevalent throughout the database.

A total of 123 participants are represented in the dataset. Only 327 out of 593 recordings are labelled; with a 10-pixel black "header" printed at the top of each image. The images were originally of $640 \times 490$ pixels. Furthermore, the database was a mixture of 1,758 grey scaled and 204 coloured images.

\section{B. Modification of the Database}

The Cohn-Kanade AU-Coded Expression Database was modified further to give better accuracies and to achieve precision in the output.

While each sequence in the labelled set contained an approximate of 10 images depicting a transition of emotion from neutral to the peak of that affect, we decided to exclude the first few images as the emotions depicted in them were imperceptible. In aggregate, the dataset was a total of 2,600 images, representing 327 recordings. The dataset is visualised in Figure 3.2.

In preprocessing the $640 \times 10$ pixel "header" regions on top of the file was eliminated. In order to maintain uniformity in the size and colour of the database, the cascade object detector in MATLAB which uses the Viola-Jones algorithm was incorporated to identify the face in the images for cropping and resizing. This dataset was further rescaled to an array with $256 \times 256$, so as to have the same dimensions across input data. Further on, they were converted to 8-bit grey-scale values and are in tif format. (Fig. 1)
The final database trains the CNN with a seven-classes that exhibit semantic and appearance diversity. The emotions considered here are happiness, sadness, surprise, anger, contempt, fear and disgust. There are 432 images under 'Anger', 615 images under 'happiness', 320 images under 'sadness', 540 images under 'surprised', 327 images under 'fear', 116 images under 'contempt' and 340 images under 'disgust'. The final database thus contains 2600 images.

Finally, the images in the database were divided into a training set and a testing (validation) set. For each label, $80 \%$ of the images contained within it were used as the training set and the remainder served as the testing set. The images were selected at random.

\section{TECHNICAL APPROACH}

We chose to work on Jupyter Notebook 5.5.0 using Anaconda Navigator 1.8.7, opting for Keras as our framework to train the neural networks on. Keras is a scientific, userfriendly, modular, and extensible computing framework with wide support for deep neural network algorithms.

Each of our models were trained on macOS High Sierra Version 10.13.6 (17G65). This configuration provided us a platform to experiment with a variety of model configurations and specifications; however, computation speed was constrained since we were not using GPUs.

\section{TRANSFER LEARNING}

Given the complexity of deep convolutional neural networks, a popular technique called Transfer Learning is used. It attempts to transfer knowledge acquired from a particular source task and apply it to enhance learning in a similar target task. Hence, Transfer Learning can be described as "the reuse of a pre-trained model on a new problem." [4]

Due to the considerably small size of the CK+ Dataset, Transfer Learning seems like a rather appealing approach to train the network with: The features can be extracted from deeper neural networks and thereby be used to train the fullyconnected layers atop it, rather than training a deep neural network on such a small data and miserably overfitting . [5]

Here we train using the following pre-trained deeper networks:

\section{A. VGG16:}

This network is the Visual Geometry Group's implementation of deep convoluted neural network. It has 13 convolutional layers and three fully-connected layers that enumerates an input of $227 \times 227$ pixel face image. It is an integration of five convolutional layers that maintains a filter size with a very small receptive field of $(3 \times 3)$, in order to capture every pixel from the input image. The convolution stride is fixed to 1 pixel, while spatial pooling is carried out by 5 max-pooling layers, by preserving the spatial resolution after convolution by padding the image, i.e. the padding is 1 pixel for $(3 \times 3)$ convolutional layers. Max-pooling is performed over a $2 \times 2$ pixel window, with stride 2 . [6][7]

The series of convolutional layer is further trailed by three Fully- Connected (FC) layers: each of the initial two consisting of 4096 channels each, while the last one performs 
a 1000- way ILSVRC classification. The soft-max layer finally concludes the network. All hidden layers are equipped with ReLU.[7]

The VGG16 architecture has a total of $134,289,223$ parameters of which all are trainable parameters. It can train with 1000 distinct classes at a time.

To accommodate the network to our necessities, the last layer (FC layer of size 1000 with Softmax activation) of this architecture, was modified and changed to the size of 7 from size 1000 , as we have seven distinct emotion classes specified in our database.

The weight of the pre-trained model is $528 \mathrm{MB}$, providing a Top-1 Accuracy of $70.5 \%$ and Top-5 Accuracy of $90.0 \%$. [6][7]

\section{B. ResNet-50:}

The network comprises of a convolutional layer with 64 filters and a kernel size of $7 \times 7$ followed by a max pooling layer of $(3 \times 3)$ with stride specified as 2 in both cases. The architecture is then succeeded by four more convolutional layer, each of whose layers are grouped in trios, with alternating kernel sizes of $(1 \times 1)$ and $(3 \times 3)$, with a fixed feature map dimension [64, 128, 256, 512] respectively, bypassing the input every 2 convolutions.. The following four convolutional layers reports itself 3, 4, 6 and 3 times respectively. The final layer is the average pool layer with soft-max activation consisting of 1000 channels, one for each class.

On observing the first operation of each layer, we notice that the stride utilised for the introductory layer is 2 , unlike the remaining (with stride:1). Hence, the ResNet network, increases the stride to down-sample the volume, unlike the regular CNNs that make use of a pooling layer to perform the task. Moreover, only one max pooling operation is performed in this Convolutional layer, and one average pooling layer at the end of the ResNet, right before the fully connected dense layer. [8]

The ResNet50 architecture has a total of $23,602,055$ parameters of which $23,548,935$ are trainable while the remaining 53,120 are non-trainable parameters. It can train with 1000 distinct classes at a time. To accommodate the network to our necessities, the last layer (Average pooling layer of size 1000 with Softmax activation) of this architecture, was modified and changed to the size of 7 from size 1000 , as we have seven distinct emotion classes specified in our database.

\section{SQUEEZE AND EXCITATION APPROACH}

This architecture was proposed by researchers from the Oxford university[12]. The method focuses on enhancing the channel interdependencies while maintaining the computational cost to it's minimum. This allows us to adaptively recalibrate the Channel-wise feature maps while boosting the significant features and suppressing the rest, simultaneously, by explicitly modelling the interdependencies between channels. [9]

While the integration of the Squeeze and Excitation Network into a desired architecture can be implemented in several ways (Concurrent channel squeeze \& excitation (SE), Squeezing spatially and exciting channel-wise (cSE),
Squeezing channel-wise and exciting spatially (sSE), Concurrent spatial and channel squeeze \& excitation (scSE)), the generic method involves an introduction of a function command with an input convolutional block and the number of channels it contains. Each channel is then squeezed to a single numeric value using average pooling. A set of Fully Connected (FC) layers concludes the network: the initial one along with the ReLU function introduces the necessary nonlinearity; while the latter one provides each channel with a smooth gating function by sigmoid activation. [10]

We made use of the SE Standard Block and SE Identity Block in the ResNet-50 architecture to get a total of $26,133,047$ parameters of which $26,079,927$ are trainable while the remaining 53,120 are non-trainable parameters. Every SE block in ResNet-50 operates on a global average pooling operation in the squeeze phase and two small fully connected layers in the excitation phase, followed by an inexpensive channel-wise scaling operation.

\section{Results}

\begin{tabular}{|l|l|l|l}
\multicolumn{1}{|c|}{ PARAMETERS } & \multicolumn{1}{|c|}{ SE-RESNET50 } & \multicolumn{1}{c|}{ VGG16 } & \multicolumn{1}{c}{ RESNET-50 } \\
\hline $\begin{array}{l}\text { Training Accu- } \\
\text { racy }\end{array}$ & $100 \%$ & $99.95 \%$ & $99.95 \%$ \\
\hline $\begin{array}{l}\text { Validation } \\
\text { Accuracy }\end{array}$ & $99.36 \%$ & $98.07 \%$ & $37.56 \%$ \\
\hline $\begin{array}{l}\text { Time Taken for } \\
\text { 25 Epochs }\end{array}$ & 12 hours & $\begin{array}{l}8 \text { hours } 19 \\
\text { minutes }\end{array}$ & $\begin{array}{l}4 \text { hours } 49 \\
\text { minutes }\end{array}$ \\
\hline Precision Value & 0.99 & 0.98 & 0.68 \\
\hline Recall Values & 0.99 & 0.98 & 0.38 \\
\hline F1-Score & 0.99 & 0.98 & 0.40 \\
\hline Support & 780 & 780 & 780 \\
\hline \multicolumn{4}{|c}{ RESULT ANALYSIS } \\
\hline
\end{tabular}

Table 1 . The table shows a comparative study of different parameters between the models used (Squeeze and Excitation Network in Resnet50, VGG16, ResNet-50)

To maintain consistency in each of the models, the same image dataset was used. A uniformity was maintained in the training procedures while the dataset was shuffled randomly at a constant $4: 1$ proportion, thereby using $80 \%$ of the images for training and the remaining $20 \%$ for validation purposes. To further standardise the output, 25 epochs were computed for each of the networks, while saving full run history and the weights of the model in $\mathrm{H} 5$ format.

\section{A. $\mathrm{VGG16}$}

The training options specified were 25 maximum epochs, a mini-batch size of 256 , a stochastic gradient descent momentum parameter of 0.9 , initial learning rate of 0.001 , and a validation frequency of 5 . The model was trained with an accuracy of $99.95 \%$, giving a validation accuracy of $98.07 \%$, taking approximately 8.5 hours to train. The confusion matrix is shown in fig. 2. 


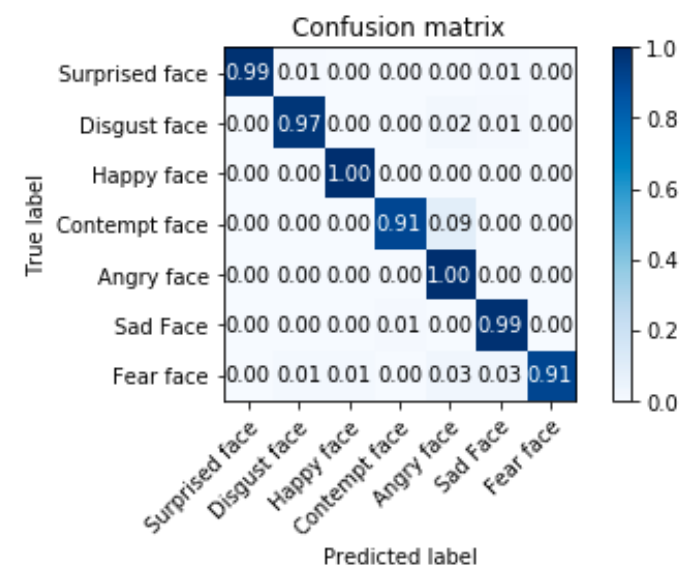

Fig. 2. The confusion matrix obtained after training on VGG16 Architecture

\section{B. ResNet-50}

The training options specified were 25 maximum epochs, a mini-batch size of 256 , stochastic gradient descent momentum parameter of 0.9 , initial learning rate of 0.001 , and a validation frequency of 5 . The model was trained with an accuracy of $99.95 \%$, giving a validation accuracy of $37.56 \%$, taking approximately 5 hours to train. The confusion matrix is shown in fig. 3 .

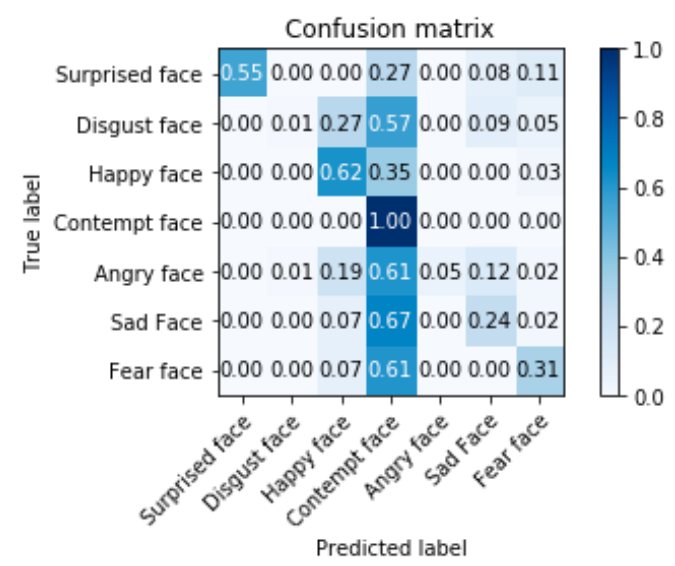

Fig. 3. The confusion matrix obtained after training on ResNet-50 Architecture

\section{Squeeze and Excitation ResNet-50}

The training options specified were 25 maximum epochs, a mini-batch size of 256 , stochastic gradient descent momentum parameter of 0.9 , initial learning rate of 0.001 , and a validation frequency of 5. The model was trained with an accuracy of $100 \%$, giving a validation accuracy of $99.36 \%$, taking approximately 12.5 hours to train. The confusion matrix is shown in fig. 4.

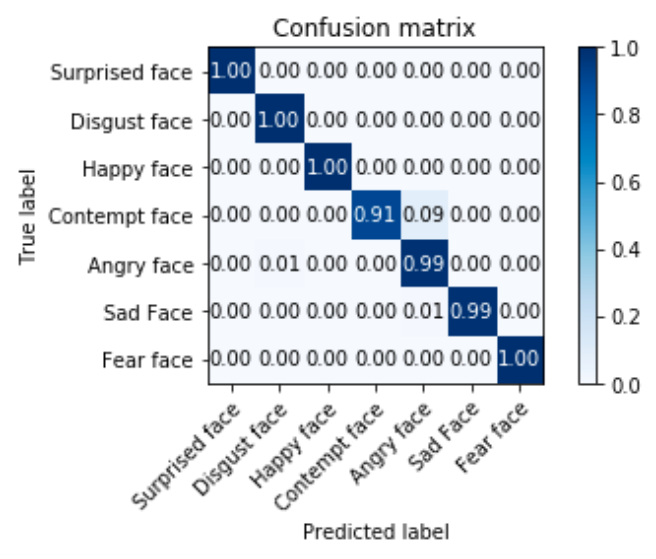

Fig. 4. The confusion matrix obtained after incorporating the squeeze and excitation approach to the ResNet-50 Architecture

We observed that in the final layers of the network where prominent diversity of representation exists within a single class, the network utilises feature recalibration to upgrade its discriminative performance. In conclusion, SE blocks generate instance-specific responses which thereby bolsters the ceaseless needs of class-specific models at different layers in the architecture.

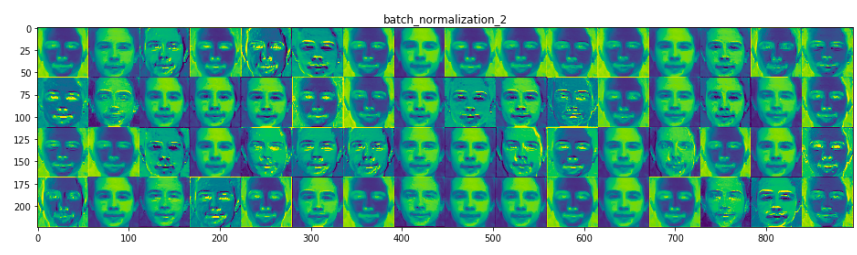

Fig. 5. Features extracted after the batch normalisation of the final channel on a randomly picked image

\section{CONClusion}

On examination, the results showed that Squeeze and Excitation Network ResNet-50 architecture achieved accuracies that were comparable to that of VGG16 network, of about $98-99 \%$ validation accuracy.

To further evaluate the results, a comprehensive analysis was done using the classification report of the three networks. We found that the precision and recall values for the above two networks provided excellent, almost equivalent results, while the ResNet-50 model provided quite different results, revealing poor accuracies and less efficiency, for our model.

In addition to the accuracies, the loss values seemed to decrease to negligible for the SE- ResNet-50 model and VGG16 model while the loss values remained constant for the ResNet-50 model.

\section{REFERENCES}

1. Picard, R.W., 2000. Affective computing. MIT press.

2. FP. Ekman and W.V. Friesen, "Manual for the Facial Action Coding System," Consulting Psychologists Press, 1977.

3. Lucey, P., Cohn, J.F., Kanade, T., Saragih, J., Ambadar, Z. and Matthews, I., 2010, June. The extended cohn-kanade dataset (ck+): A 
complete dataset for action unit and emotion-specified expression. In 2010 IEEE Computer Society Conference on Computer Vision and Pattern Recognition-Workshops (pp. 94-101). IEEE.

4. https://builtin.com/data-science/transfer-learning

5. Wang, S., 2016. Facial affect detection using convolutional neural networks. Stanford University.

6. https://medium.com/@RaghavPrabhu/cnn-architectures-lenet-alexnetvgg-googlenet-and-resnet-7c81c017b848

7. https://neurohive.io/en/popular-networks/vgg16/

8. http://pabloruizruiz10.com/resources/CNNs/ResNets.pdf

9. https://towardsdatascience.com/squeeze-and-excitation-networks-9ef5e71 eacd7

10. Roy, A.G., Navab, N. and Wachinger, C., 2018, September. Concurrent spatial and channel 'squeeze \& excitation'in fully convolutional networks. In International Conference on Medical Image Computing and Computer-Assisted Intervention (pp. 421-429). Springer, Cham.

11. Reisenzein, R., 2010. Broadening the scope of affect detection research. IEEE Transactions on Affective Computing, 1(1), pp.42-45.

12. Hu, J., Shen, L. and Sun, G., 2018. Squeeze-and-excitation networks. In Proceedings of the IEEE conference on computer vision and pattern recognition (pp. 7132-7141). 